\title{
Educación musical, desarrollo infantil y adolescente y enfoque de derechos humanos: Una reseña bibliográfica
}

\section{Musical Education, Child And Adolescent Development, And Human Rights Perspective: A Bibliographic Review}

\author{
Consuelo Arguedas-Quesada \\ Universidad de Costa Rica \\ Escuela de Formación Docente \\ Instituto de Investigación en Educación, INIE \\ San José, Costa Rica \\ consueloaq@gmail.com
}

Recibido: 22 octubre 2013 Aceptado: 4 diciembre 2014 Corregido: 11 mayo 2015

Resumen: El siguiente documento surge de la indagación bibliográfica que realiza la autora de este artículo, para llevar a cabo su investigación en la Maestría Profesional en Derechos Humanos de la Niñez y la Adolescencia, del Programa de Estudios de Posgrado en Trabajo Social de la Universidad de Costa Rica. Para tal efecto, hace una revisión sobre los estudios relacionados con la temática a nivel nacional e internacional al momento de la investigación que abarca los siguientes tópicos: práctica pedagógica musical, educación musical y currículo escolar, música y sindrome de Down, inteligencias múltiples y desarrollo integral, expresión artística y desarrollo humano, enfoque de derechos y expresión artística, y los derechos humanos de las personas con discapacidad. El objetivo del estudio tiene como propósito comparar y contrastar dichos temas, vinculándolos con los derechos humanos, la educación musical, y su práctica en personas en condiciones asociadas o no con la discapacidad. La finalidad es compartir estos hallazgos con personas interesadas en esta temática. Como parte de las conclusiones, la búsqueda muestra que en Costa Rica no existen trabajos de educación musical relacionados con derechos humanos de la niñez y la adolescencia y, en la mayoría de los trabajos, la música se plantea desde las inteligencias múltiples.

Palabras clave: Educación musical, derechos humanos, niñez y adolescencia, desarrollo humano, discapacidad.

Abstract: This document emerges as a result of the bibliographic review performed by the author in order to achieve her Master degree in Human Rights of Children and Adolescents, for the Graduate Program in Social Work at the University of Costa Rica. For that reason, the article reviews several studies performed by the time of the investigation based on the national and international background knowledge. The following topics are discussed: pedagogic musical practice, musical education and school curriculum, music and Down' syndrome, multiple intelligence and integral development, art expression and human development, rights and art expression, and Human Rights for disabled people. The objective of this study is to compare and contrast those subjects, allowing the linking of Human Rights to musical education and its practice in disable people or not, with the objective of sharing the results with people interested in this area. As part of the conclusions this search shows that in Costa Rica there are no jobs related to Human Rights and musical education for Children and Adolescents, and most music works are focused on the multiple intelligences.

Key Words: Musical Education, Human Rights, childhood and adolescence, human development, disability.

1 Magister en Derechos Humanos de la Niñez y la Adolescencia. Profesora de educación musical y expresión corporal en la Escuela de Formación Docente de la Facultad de Educación, e investigadora del Instituto de Investigación en Educación (INIE) de la Universidad de Costa Rica. Dirección electrónica: consuelo.arguedas@ucr.ac.cr 
Este artículo surge de la investigación de la autora: Análisis del modelo pedagógico musical que utilizan los Programas Especiales del Sistema Nacional de Educación Musical del Ministerio de Cultura y Juventud, en relación con las necesidades e intereses de sus estudiantes desde el enfoque de derechos humanos. Esta se realizó en la Maestría en Derechos Humanos de la Niñez y la Adolescencia, perteneciente al Programa de Estudios de Posgrado en Trabajo Social de la Universidad de Costa Rica.

Con base en esta investigación, la autora de este artículo realiza una búsqueda de documentos de grado y posgrado, u otros estudios originados de tópicos similares llevados a cabo en Costa Rica y en otros países, los cuales se convierten en insumos para la elaboración del presente documento. Debido a que en nuestro país hay poca información al respecto, se persigue que este texto sirva de plataforma para que otras personas interesadas en la temática continúen indagando sobre ella y, de esta manera, se reivindique la educación musical de la niñez y de la adolescencia, en beneficio de su desarrollo integral y como parte de la legislación establecida en derechos humanos.

Las indagaciones que conforman el sustento teórico del artículo se organizan desde el contexto nacional e internacional, y se organizan según los tópicos tratados.

\section{Principales temas encontrados a nivel nacional}

\section{Práctica pedagógica musical}

Para abordar este componente, el artículo de Arguedas (2006) plantea que la educación musical ofrece múltiples posibilidades para "hacer música", vivenciarla e interiorizarla. Su enseñanza y aprendizaje no se basa solo en aspectos teóricos, memorísticos o repetitivos; por el contrario, las diversas áreas de la educación musical permiten incursionar de manera activa, según la experiencia de cada persona, estimulando la libre expresión y las posibilidades sonoras del cuerpo. Es decir, "saber música" engloba un enfoque amplio, donde la población estudiantil tiene acceso a todo tipo de músicas, puede experimentar con los sonidos y los ruidos y, por ende, con el empleo de grafías no convencionales, así como también con la expresión rítmica, melódica, armónica, instrumental, vocal y corporal. La relación que se establece en la niñez entre los objetos y el juego, ya sea individualmente o con otros niños y niñas, convierte al sonido en un puente entre la fantasía y la realidad.

Arguedas (2006) ofrece insumos a partir de la necesidad de interiorizar la expresión musical, para estimular las áreas de la audición, la rítmica, el canto, la lecto-escritura, la ejecución instrumental y la apreciación musical, mediante planteamientos interesantes; es decir, vivenciar la música equivale a hacerla propia desde diversas estrategias metodológicas.

\section{Educación musical y currículo escolar}

Como parte de esta modalidad se rescata el documento de Bonilla, García y Ledezma (2001), cuyo objetivo consiste en analizar cómo se organizan el aprendizaje en torno a la 
educación musical y la expresión corporal, en los centros educativos del ciclo de transición de la educación inicial.

Además, en él se proponen lineamientos metodológicos que se incorporan a una propuesta didáctica, en correlación con los bloques temáticos del currículo de preescolar, establecidos por el Ministerio de Educación Pública de Costa Rica.

Por su parte, Arguedas (2003) presenta una reflexión sobre la necesidad e importancia de que el personal docente estimule la improvisación musical ${ }^{2}$ en la población escolar, ya que con ella se facilita la puesta en práctica de contenidos de las diferentes áreas de la educación primaria, mediante estrategias didácticas dinámicas y placenteras que favorecen los procesos de enseñanza y aprendizaje. Asimismo, se convierte en un elemento didáctico de gran importancia para el desarrollo cognitivo, afectivo y psicomotor que estimula la creatividad, la originalidad y espontaneidad innatas de la niñez y de las personas adolescentes.

De igual manera Arguedas (2004b) describe los beneficios de la expresión musical, sus características y objetivos, resaltando la importancia de integrarla en el currículo escolar desde una perspectiva integral del ser humano. Además Arguedas (2009), en su trabajo investigativo, plantea la concepción de la música como un lenguaje que le permite a la población estudiantil disfrutar de ella ampliamente, como un derecho a manifestar sus sentimientos, emociones y estados de ánimo de manera creativa y artística, con la finalidad de que logren conmoverse y conmover a las demás personas. Esta es una tarea del cuerpo docente, un compromiso que debe cumplirse desde la integralidad curricular, pues los niños, las niñas y las personas adolescentes requieren una formación holística.

De la misma manera, Jiménez (2009) plantea una propuesta metodológica articulada al currículo preescolar, en la cual se realizan talleres a docentes para capacitarlas en diversas áreas de la educación musical. Posteriormente estas maestras aplican los conocimientos recibidos a sus estudiantes en los respectivos centros educativos donde laboran, como evidencia de los logros obtenidos en el proceso.

\section{Música y síndrome de Down ${ }^{3}$}

Es importante indicar que en la búsqueda que se realiza a nivel nacional existen indagaciones en relación con síndrome de Down; no obstante, el componente de educación musical es limitado y aparece con la denominación de musicoterapia. Al respecto, se revisa el trabajo de Mora (2001), cuyo propósito consiste en determinar los efectos agudo y crónico de la musicoterapia y la danzaterapia en el comportamiento no verbal en personas con síndrome de Down y discapacidad múltiple severa.

El proyecto es un estudio piloto que aplica el diseño experimental, donde el grupo participante es de sexo femenino y masculino, el cual es dividido en cuatro grupos: músicadanza, música, danza y grupo control. Los grupos experimentales reciben tres sesiones

\footnotetext{
2 La improvisación musical no es sinónimo de caos, por el contrario, se basa en estructuras rítmicas, melódicas, consignas o de otra índole sonora previamente establecidas por el personal docente, que sirven de plataforma para que la población estudiantil a partir de ella, juegue, invente, transforme y exprese su creatividad haciendo uso de la altura, el timbre, la intensidad, la velocidad, la duración, la textura u otros componentes del sonido.

3 Se incluye el síndrome de Down, pues forma parte de la población participante en el proyecto investigativo que da origen a este artículo.
} 
semanales de veinte minutos cada una durante siete semanas; utiliza un pretest y un postest con las variables de contacto visual, sonrisa y vocalizaciones. La investigación demuestra superioridad en el grupo música-danza con respecto a los otros dos, mientras que el grupo control no muestra cambios en las mediciones.

\section{Inteligencias múltiples y desarrollo integral}

Al respecto, Chavarría y Orozco (2006) destacan las ventajas del ecoanálisis como andamiaje para decodificar los valores que inconscientemente se plasman en las relaciones y ambientes que se preparan para la formación de la niñez preescolar, mediante diálogos concretos. Chavarría y Orozco (2006, p. 14) definen dicho ecoanálisis como el "análisis de los espacios y situaciones que conforman nuestro acontecer cotidiano, el cual nos permite la decodificación para facilitar cambios en las relaciones entre las personas y sus mundos, implica mirar y analizar con ojo crítico el diálogo yo-mundo que se da en las rutinas diarias de un centro infantil". Es así como se promueven acercamientos progresivos y compartidos hacia cosmovisiones acordes con la realidad actual, potenciando lo cotidiano y las transformaciones curriculares desde comunidades de aprendizaje transdisciplinarias.

Además, mediante el ecoanálisis se producen modificaciones significativas en el quehacer infantil, se refuerza el holismo, el enfoque de derechos y la interiorizan de valores plasmados en lo cotidiano, a partir de la integración cuerpo-emociones-mente. Dicho estudio traza aspectos de una educación inicial transformadora, en donde el entorno, mediatizado por lo cultural, delimita y ofrece o resta posibilidades. Por este motivo, las autoras plantean que solo cuando se comprende que la realidad es producto de costumbres aprendidas se logra re-aprender y visualizar otras posibilidades, las cuales propician la innovación de la cultura hacia valores y acciones imprescindibles para el momento presente.

Otra autora que hace referencia a esta temática es Carballo (2006), quien relata lineamientos de la educación de la inteligencia emocional y la educación de la expresión de la sexualidad humana, tomando como base los planteamientos de Howard Gardner y Daniel Goleman. Ella hace énfasis en la importancia de la expresión de las emociones en la niñez y la adolescencia, sin hacer ninguna distinción de género. De igual forma Carballo (2006) alude a los talentos que posee el ser humano y que expone Gardner, los cuales se agrupan en siete inteligencias: lógico matemática, musical, lingüística, espacial, cinestésica, intrapersonal e interpersonal; aunque considera que existen otras como la espiritual, que se basa en los principios éticos de la persona, la subjetividad y el desarrollo de la sensibilidad estética. El crecimiento de cada una de ellas depende de las acciones recíprocas de la herencia biológica, las prácticas de crianza en la educación y el contexto histórico cultural que se viva. Asimismo se comenta sobre la reflexión de Goleman relacionada con la inteligencia intrapersonal e interpersonal en el desarrollo humano, las cuales denomina inteligencia emocional.

Fernández (2007), por su parte, lleva a cabo una investigación desde el enfoque cualitativo, que se orienta hacia la satisfacción de las necesidades de la sobrevivencia como elementos necesarios en la formación de una sociedad solidaria y tolerante. Considera que el término necesidades educativas especiales marca discapacidades y pobreza, estigmatizando estas 
poblaciones, limitándolas y marginándolas en su crecimiento profesional. De igual manera opina que en Costa Rica el personal docente que atiende a las personas talentosas necesita recursos y mayor atención profesional para brindarles mejores lineamientos metodológicos.

Este trabajo enfatiza en la gama de potencialidades y capacidades que poseen las personas (dentro de las cuales está la expresión musical), las cuales pueden alcanzar grandes logros si son orientadas de manera profesional. Se fundamenta en la inteligencia emocional e indica la importancia de regular los propios sentimientos, comprender las emociones de los demás, trabajar con otras personas y sentir empatía por ellas (Fernández, 2007).

\section{Expresión artística y desarrollo humano}

Dentro de esta categoría se revisa la tesis de maestría de Poltronieri (1998), la cual se orienta a recuperar el sentido de una educación para preescolares con carácter descolarizante, a partir de la cotidianidad en las experiencias, y respetando el ritmo y sus necesidades en el proceso de aprendizaje. Se fomenta la exploración, la independencia y el interés por participar en actividades con integración de género, y el respeto a la naturaleza en la construcción de valores. Se tienen como punto de partida los principios montessorianos, que se enriquecen con el análisis de corrientes interactivas del desarrollo del niño y la niña, las propuestas del modelo de currículo de marco abierto, aportes de la pedagogía crítica y la metodología de ecoanálisis.

Otro trabajo investigativo es el de Montoya y Sánchez (2000). En él se presenta la expresión corporal como una propuesta para estimular la creatividad, como parte de la expresión artística de la población preescolar. Además, realza la labor de aula y brinda sugerencias prácticas basadas en la teoría respetando las diferencias individuales, las cuales se asocian a las necesidades e intereses estudiantiles particulares y, con ello, al disfrute de sus derechos.

Por otra parte, Arguedas (2004a) destaca el valor didáctico de la expresión corporal, integrada con el currículo escolar de forma transversal. El tema se deriva del proyecto de investigación de la autora denominado, La expresión artística: Un recurso heurístico para la construcción de procesos pedagógicos desde una perspectiva de género (García, Castro, Arguedas, 2003), el cual se realizó en una escuela de atención prioritaria. Es así que se indica que la expresión corporal no requiere aptitudes especiales; por el contrario, el ser humano necesita de ella sin excepción. Es el medio idóneo para promover el desarrollo intelectual, físico, sensorial y emocional, con técnicas que favorecen a la vez la atención, la concentración y el elemento creativo; aspectos necesarios también en las diversas áreas del conocimiento. La expresión corporal logra integrar los contenidos curriculares y evita una educación repetitiva e indiferente, al emplearse como eje transversal en el proceso educativo. Los temas más diversos resultan ser de gran interés para el estudiantado, al proporcionárselos el personal docente en forma lúdica y no tradicional; como consecuencia, la expresión corporal enriquece la labor pedagógica y el desarrollo integral de la población estudiantil.

Otro trabajo que ofrece datos en relación con el tópico a tratar es el de Arguedas, Castro y García (2006), el cual constituye un material que permite innovar las prácticas pedagógicas que se desarrollan en los centros educativos. Desde un enfoque humanista que incorpora el holismo y el constructivismo en la educación, se enfatiza en la importancia de trabajar, de 
manera integrada, las artes plásticas, la música y la expresión corporal al contenido escolar, con el propósito de que la población estudiantil construya y aplique su aprendizaje en el contexto natural y sociocultural en donde se desenvuelve.

Es así como se ofrecen sugerencias en aras de propiciar un ambiente de aprendizaje que permita el desempeño creativo y con expresividad de la población estudiantil, desde el punto de vista cognitivo, psicomotor y socioafectivo. La propuesta de investigación emerge de una realidad educativa denominada "Aula abierta", y ofrece planteamientos significativos mediante la planificación de actividades lúdicas.

Otro aporte sobre la temática en cuestión lo brindan Arguedas y Castro (2009), mediante ejemplos contextualizados en la lógica de planeamiento desde la integración de la matemática, ciencias, estudios sociales y español con la expresión musical, corporal y plástica, para ser aplicados en procesos educativos formales, no formales e informales. Dicha proposición se convierte en un material de estudio para la población docente, pues pretende transformar las prácticas de aula en experiencias agradables y significativas para la población escolar, desde una perspectiva construccionista y holista.

\section{Enfoque de derechos y expresión artística}

Como nota interesante se aclara que no se encontró información concerniente con esta categoría a nivel nacional (y de manera especial en relación con la enseñanza de la música, como parte del interés temático de la investigación). No obstante, Montero (2004) argumenta, en un artículo, sobre las múltiples carencias legales, políticas e institucionales para el desarrollo de políticas públicas en el campo de la cultura, tanto en el ámbito internacional como nacional. Como consecuencia, el investigador inicia su trabajo con un acercamiento al concepto de derecho cultural, para luego explorar su desarrollo histórico y su contenido programático actual. Posteriormente, el análisis se centra en las limitaciones normativas, políticas, administrativas y conceptuales de los derechos culturales que han restringido su exigibilidad y vigilancia en el cumplimiento por parte de las Naciones Unidas hacia los Estados miembros que han ratificado estos derechos.

\section{Los derechos humanos (DDHH) de las personas con discapacidad}

En relación con esta temática, Herrera (2002) hace una reseña crítica de la legislación sobre la discapacidad a nivel mundial y nacional, e informa sobre los derechos humanos de estas personas. Realiza un recuento de cómo esta población a través de la historia ha vivido el aislamiento y hasta se le ha eliminado, pero con el correr del tiempo la desigualdad en el trato se modifica al pasar de tener un derecho a la sobrevivencia, luego a la dependencia y, por último, a su vida independiente a partir del nuevo paradigma que se planeta en 1980.

Indica que en un inicio se tomaban medidas paliativas, que consideraban a estas personas como el problema, sin tener en cuenta sus potencialidades ni las deficiencias de la sociedad. Por el contrario, hoy se hace énfasis en el respeto y la integridad brindando nuevas opciones sociales en el quehacer cotidiano, ya que asumen responsabilidades en relación con su vida, 
a nivel familiar y desde su comunidad. En este sentido se menciona que las personas con discapacidad no son el problema, sino el entorno es el que no reúne las condiciones necesarias para solventar sus necesidades, y de esta manera se irrespetan sus derechos humanos.

Herrera (2002) comenta que a partir de la Declaración Universal de los Derechos Humanos DUDH, la ONU ha promovido numerosa legislación en la igualdad de oportunidades en todos los ámbitos para las personas con discapacidad, nombrando la Declaración de los Derechos del Deficiente Mental (1971), la Declaración de los Derechos de los Minusválidos (1975), las Normas Uniformes sobre la Igualdad de Oportunidades para las Personas con Discapacidad (1993), el Marco de Acción sobre Necesidades Educativas Especiales (1994), la Convención Interamericana para la Eliminación de todas las formas de Discriminación contra las personas con Discapacidad (1999) y, en nuestro país, hace referencia a la Ley sobre Igualdad de Oportunidades para las Personas con Discapacidad en Costa Rica, Ley 7600 (1996).

\section{Principales temas encontrados a nivel internacional}

Por otra parte, además de la información mencionada a nivel nacional, se obtienen documentos de otros países que permiten acrecentar la búsqueda, y de igual manera, clasificar los hallazgos a partir de las temáticas antes establecidas.

\section{Práctica pedagógica musical}

En esta categoría se revisa el trabajo de Delgado (2008), en el cual se reflexiona sobre la importancia de la educación musical en la educación primaria y en la educación en general, y su influencia en el desarrollo integral de la persona. Parte de que la música es una terapia para las enfermedades tanto psicológicas como corporales, perfecciona los sentidos y el desarrollo motor, y se convierte en un vehículo para la comprensión de la cultura, la historia, la creatividad y la sensibilidad musical.

\section{Educación musical y currículo escolar}

En este tópico se recupera la labor de Sihana y Pechin (2002), quienes investigan la formación docente de las áreas de educación física y educación musical. El estudio arroja datos sobre posibilidades y límites (ideológicos cognitivos, informativos, afectivos) de la intervención educativa desde estos saberes en nivel inicial y primero y segundo ciclo de la educación general básica. De igual manera, se enfatiza en el interés de avanzar en un conocimiento y una práctica que comience a romper con los estereotipos existentes y proponga opciones significativas para las personas involucradas en dichos procesos, propiciando la interiorización y vivencia de contenidos y, con ello, una formación musical de calidad, que incluya no solo aspectos teóricos, sino el aprendizaje de un instrumento y la práctica en talleres y grupos musicales.

Por su parte, Cantón y Fabián (2010, p. 109) realizan un análisis del currículo musical en el aula de educación primaria, y consideran que este está orientado hacia el análisis de 
los programas de estudio, pero también hacia la experiencia aplicada en el aula, mediante la ejecución, la audición y la composición; y lo justifican al considerar que el conocimiento en el campo del arte involucra a la persona en experiencias en las que participa como espectadora o a partir de la experiencia.

A propósito de la temática, realizan estudios para evaluar, conocer y mejorar el estado y desarrollo del currículo en relación con la educación musical escolar en Argentina, e indican que hacen falta estrategias metodológicas adecuadas, materiales y recursos que permitan proyectos formativos y creativos. Esto, debido a que parte de la población docente no posee herramientas para el proceso de enseñanza y, como consecuencia, el aprendizaje musical escolar solo es un espacio para quienes tienen talento, y no para la participación de la población estudiantil en general.

Otra experiencia interesante es la de Palacios (2006). Opina que la música hace alusión a la mente, al cuerpo y a las emociones al impulsar procesos de intuición, percepción, simbolización, abstracción y afectividad. No obstante, cree que está muy lejos de ser realidad, la idea de considerar el potencial formativo de la música y el arte en todas sus expresiones en los centros educativos. Palacios (2006) comenta que se sigue dando prioridad al estímulo lingüístico y lógico-matemático, limitando otras áreas del desarrollo del niño y la niña, y a la vez considerando la educación artística como un fenómeno aislado, y no como aquella que forma parte del contexto de la educación en general.

Otro aporte relacionado con este tema lo brinda Rusinek (2004), quien plantea que el aprendizaje musical demanda el desarrollo de habilidades específicas de audición, ejecución y creación, y se apoya en la asimilación de contenidos y en la práctica. Parte de los planteamientos de Hanesian, Ausubel y Novak (1978) los cuales indican que en el aprendizaje la nueva información resulta significativa, si se relaciona con lo que la persona ya sabe. Consecuentemente, opina que los conceptos musicales se aprenden combinando los ejes recepción-descubrimiento y lo significativo- memorístico.

Por lo tanto, Rusinek (2004) considera que existen dos perspectivas del aprendizaje musical significativo: la primera ocurre cuando se da una relación inductiva o deductiva con la experiencia musical vivida; la segunda opción se relaciona con los significados atribuidos a la experiencia musical como procedimientos de aprendizaje; es decir, desde la voluntad de las personas, lo que las motiva para que se realice el aprendizaje. Por otra parte, Rusinek (2004) menciona que para comprender los problemas de la educación musical es necesario conformar equipos que permitan la interpretación colaborativa, intercultural e interdisciplinar, teniendo como punto de partida la preocupación por debatir lo que significa el conocimiento musical, pues opina que las desavenencias epistemológicas han causado grandes confusiones curriculares entre profesionales de la musicología, la educación musical e instrumentistas. Además, cree necesario revisar el conocimiento procedimental y los criterios de evaluación que se ofrecen en los centros educativos, así como también elaborar una fundamentación epistemológica del modelo de docente de música con un rol en la investigación, y con formación en redes y canales para difundir sus propias innovaciones educativas.

Se considera que a partir de esta perspectiva, para llevar a cabo la educación musical con personas en condiciones asociadas o no a discapacidad, el apoyo profesional desde la interdisciplinariedad es necesario, pues además de la ejecución de un instrumento musical, es 
de suma importancia que el personal docente de educación musical se convierta en conocedor de las necesidades específicas de sus estudiantes, como parte de sus derechos.

\section{Música y síndrome de Down}

En este tópico se encontraron investigaciones desde la musicoterapia, entre ellas la de Poch (2002), en la que se menciona que, desde esta especialidad, se llevan a la práctica actividades que presentan un contenido musical para trabajar un punto específico del aprendizaje de la persona, incluyendo un aspecto motivacional para estimular el área socio afectiva, el cual, al mismo tiempo, favorece otras áreas del desarrollo como un eje transversal.

Es así que Bruscia (citado por Poch, 2002) opina que la musicoterapia es un arte que tiene que ver con subjetividad individualidad, creatividad y belleza, pues la música está compuesta por sonidos que representan una estructura, un lenguaje y una expresión artística; pero también implica una ciencia que supone objetividad, colectividad, repetición y verdad.

Por su parte, Purroy y Pérez (2002, p. 57) establecen diferencias entre la educación musical y la musicoterapia, al creer que, en esta última, la música es mediadora para producir cambios como un proceso abierto, experiencial, interactivo, sistemático y evolutivo. Asimismo con contenidos dinámicos, objetivos particulares, en donde el proceso evaluativo se considera una valoración inicial y, además, la persona musicoterapeuta establece una relación de alianza y ayuda con la otra persona.

Otro trabajo sobre la temática corresponde al que realizan Pineda y Pérez (2011), relacionado con las percepciones sobre musicoterapia que tiene el cuerpo docente de un centro para personas con síndrome de Down; esto con la finalidad de valorar su aplicación en otras instituciones educativas de Cuba. Pineda y Pérez (2011) se fundamentan en la práctica musical como la base del aprendizaje de la música, y en la implementación del juego y la expresión corporal; además, dan énfasis en el desarrollo rítmico y auditivo (en este caso se trabaja la audición vocal, instrumental e infantil y el timbre sonoro).

Según Pineda y Pérez (2011), el alumnado participante en la investigación se evalúa de manera periódica por un equipo multidisciplinario, y después de realizarse la terapia durante nueve meses, las docentes admiten avances en la totalidad de la población estudiantil relacionados con el lenguaje, la socialización, el aprendizaje y las habilidades motoras. Como consecuencia, los padres y madres de familia, así como la población docente, muestran la anuencia en mantener las prácticas de musicoterapia en la institución.

Por su parte, Lagos (2004) lleva a cabo un trabajo investigativo en donde quienes participan presentan problemas de comunicación y lenguaje, se basan más en imitar que en expresar, pero se sienten motivadas respecto al quehacer musical. Realiza sesiones de intervención que le permiten obtener logros a partir del reconocimiento de las personas. De igual manera, manifiestan avances en la expresión y creación musical; en el desarrollo de la autoestima; en las capacidades afectivas, sociales y comunicativas, y se favorece la estimulación del lenguaje y las capacidades cognitivas. Al mismo tiempo, Lagos (2004) menciona que utiliza claves comunicativas preverbales que permiten la expresión mediante el lenguaje sonoro y, así, se da énfasis en las capacidades y no en las carencias. 
En este sentido Lagos (2004) comenta que el trabajo grupal es importante para el desarrollo personal; no obstante, considera que a pesar de los logros alcanzados, es necesario ampliar la duración del proceso, trabajar con padres y madres de familia, y llevar a la práctica un trabajo interdisciplinario para enriquecer la investigación.

Gutiérrez, Ibáñez y Prieto (2011), en su proyecto, hacen énfasis en la inteligencia emocional de personas con síndrome de Down, mediante la estimulación sensorial por medio de la aplicación de la música, pues opinan que propicia diversidad de sentimientos y emociones, y potencia las habilidades sociales para tener una mejor calidad de vida.

Para conseguir los objetivos planteados en dicho proyecto, las autoras se basan en la definición de las cuatro zonas de desarrollo humano:

Zona de pensar donde intentamos desarrollar la inteligencia emocional; Zona de comunicar en la que pretendemos que aprendan a expresar los sentimientos y las emociones; Zona del amor: donde mostramos nuestros sentimientos y emociones y Zona del movimiento: también podemos expresar nuestros sentimientos que nos despierta la música a través de los movimientos corporales. (Gutiérrez, Ibáñez y Prieto 2011, p. 33)

Así, como parte de sus conclusiones, mencionan que gracias al trabajo con la música, el grupo participante en su investigación organiza sus ideas para expresarlas, se relaja, crea, realiza actividades en público y tiene más participación en la vida diaria, se optimiza la tolerancia y la empatía con sus compañeros y compañeras, y se da un mejor control de emociones; todo esto conlleva a perfeccionar el desarrollo individual y social de las personas y, por ende, su calidad de vida.

\section{Inteligencias múltiples y desarrollo integral}

Al respecto, Ferrándiz, Prieto, Bermejo y Ferrando (2006) hacen referencia a las inteligencias múltiples de Gardner, como un planteamiento sugerente, que permite cuestionar el constructo de la inteligencia más allá de lo cognitivo, propiciando la capacidad de resolver problemas o de crear productos que sean valiosos en uno o más ambientes culturales.

Además, Ferrándiz, Prieto, Bermejo y Ferrando (2006) reconocen la existencia de las ocho inteligencias diferentes e independientes, que pueden interactuar y potenciarse recíprocamente, haciendo énfasis en la musical, la corporal, la interpersonal, la intrapersonal y la naturalista. La musical la asocian con la capacidad para producir y apreciar el tono, ritmo y timbre de la música, la cual se expresa en el canto, la composición, la dirección orquestal o la apreciación musical. La corporal la consideran como la capacidad para utilizar el cuerpo de manera total o parcial, manipular objetos y lograr efectos en el ambiente. La inteligencia interpersonal la definen como aquella que permite entender a las demás personas y actuar en situaciones sociales, ayudando a percibir y discriminar emociones. La intrapersonal la conciben como la que le permite al ser humano comprenderse a sí mismo, reconocer sus emociones, y los motivos por los que se reacciona de un modo u otro. Y por último, se refieren a la inteligencia naturalista, como la que brinda la posibilidad de analizar las relaciones que 
existen entre varias especies o grupos de objetos y personas, así como reconocer y establecer si existen diferencias y semejanzas entre ellas.

Otro documento que se consulta es el de Luz de Luca (s. f.), el cual es producto de la labor que realiza la investigadora en una escuela rural de Argentina, donde atiende a un estudiante que ama la naturaleza, la vida y el trabajo rural, pero que enfrenta dificultades para resolver situaciones lógico-matemáticas, extraer ideas principales de un texto, leer, redactar.

Se rescata de este artículo, la posición de la autora en relación con la importancia de estimular y detectar el nivel de desarrollo de las inteligencias, y de las capacidades o fortalezas de la población infantil y de las personas adolescentes de los distintos centros educativos, ya que esto permite planificar las estrategias metodológicas acordes con sus necesidades e intereses.

\section{Expresión artística y desarrollo humano}

Como parte de esta temática, Green (2001) realiza un trabajo investigativo donde la música estimula el potencial humano, e inicia su reflexión considerando que las artes se han utilizado en individuos, comunidades y sociedades por años, pero la búsqueda en la efectividad ha sido limitada y la información que existe es fragmentada. Es así que trabaja en diferentes proyectos, los cuales consideran que el arte puede usarse a través de una amplia gama de necesidades sociales relacionadas con los derechos humanos. En ellos se recurre de manera individual y comunitaria al baile, música, literatura, artes visuales y drama, relacionados con las emociones, la percepción, la representación y la experiencia.

El trabajo concluye que las necesidades sociales que más se requieren y están relacionadas con los derechos humanos son la salud y la educación, las cuales, mediante la aplicación de las artes, solventan diversas insuficiencias, inclusive aplicándolas en otros contextos como la agricultura y la economía.

Otra investigación que se revisa es la de Sanfeliu y Caireta (2005), la cual propone una forma diferente de trabajar la educación para la paz mediante la música, pues señala que esta, además de ser un elemento de motivación, por sí misma es una herramienta pacífica, ya que provoca sensaciones y emociones, es un lenguaje de denuncias, de inquietudes personales y sociales, es un instrumento de lucha contra la no violencia, y una buena aliada para reparar conflictos. Por esta razón, Sanfeliu y Caireta (2005) plantean seis propuestas didácticas a partir de composiciones musicales, cuyos temas son los derechos humanos, los derechos de la niñez, la guerra, la paz, la migración y la diversidad. Las estrategias están planeadas para una población a partir de los diez años, e indican que, de igual manera, pueden hacerse modificaciones para la población estudiantil de secundaria, lo cual depende de la creatividad del profesorado para hacerle las adaptaciones necesarias.

Por su parte, Riera, Sansevero y Lúquez (2008) llevan a cabo un estudio que analiza la cultura democrática en la formación integral de los educandos, sustentada en una práctica pedagógica constructivista en la educación básica de Valencia, Venezuela, para fomentar el desarrollo humano. Según los resultados obtenidos, la formación que recibe el estudiantado de 
educación primaria se centra en una participación impuesta, lejos de una tolerancia recíproca que favorezca el diálogo constructivo en el aprendizaje.

En este documento se plantea que la formación integral es un reto constitucional que solo puede lograrse con estrategias democráticas, ya que mediante la educación es posible construir una cultura más participativa, representativa y respetuosa de la dignidad humana en todos los ámbitos, es decir, una cultura de la democracia, la cual debe ser vista como un estilo de vida que contribuya al desarrollo integral del alumnado desde el proceso educativo, a partir de sus necesidades e intereses y en beneficio de aprendizajes significativos.

Cabe mencionar que también se revisa el documento de Pérez (2008), el cual trata la educación como un espacio de sociabilidad de los seres humanos que merece representantes de cambio, para renovar las acciones sociales de la práctica educativa. Al mismo tiempo, la práctica pedagógica hace énfasis en la calidad humana, la mentalidad exitosa, la capacidad de trabajo y entrega, la expresión lúdico-creativa, el espíritu investigativo y al sentido de pertenencia de su entorno natural y cultural. Se rescatan de este trabajo la exploración y observación de las prácticas cotidianas de la educación artística-musical, tratando de encontrar coherencia e identidad en la manera como se abordan la didáctica, los contenidos, los sistemas de enseñanza, la evaluación, los materiales, el aprendizaje y demás mediaciones, que conlleven a establecer los fundamentos teóricos, epistemológicos, axiológicos y sociales, que sustentan la propuesta metodológica.

\section{Enfoque de derechos y expresión artística}

A propósito de este tema, en el trabajo de Olson-Horswill (2005), se comenta sobre la experiencia realizada por estudiantes universitarios de artes, con personas indigentes de un refugio y un programa no lucrativo de artes para una población infantil.

En dicha experiencia ambas poblaciones se unen para crear esculturas basadas en los 30 artículos de la Declaración de los Derechos Humanos de 1948, donde una de ellas se convierte en el símbolo de la actividad y se coloca en la biblioteca de la universidad; esta escultura consiste en una espiral de treinta manos de yeso de colores.

La investigadora opina que la persona crea, se conecta y comunica, con la finalidad de fortalecer a la comunidad por medio de su potencial; por este motivo considera que esta unificación simboliza la libertad de expresión de las personas y, por lo tanto, está articulada a los lineamientos de los Derechos Humanos.

Además, afirma que el paradigma constructivista, al igual que el proceso creativo, dan significado al estudio cualitativo, propiciando la interacción entre las personas participantes (la población adulta, artistas, grupo infantil), lo que conlleva un trabajo artístico en conjunto. Al mismo tiempo, para recoger la información, Olson-Horswill (2005) utiliza pre y pos entrevistas orales sobre las actitudes de la población estudiantil universitaria en relación con el trabajo comunal que realiza, entrevistas a niños y niñas, observaciones de las actividades, análisis de las encuestas, notas de campo y análisis de obras de arte.

Otro artículo que atañe a la temática de este apartado es el de Prieto de Pedro (2008), el cual plantea que los derechos culturales viven la paradoja de ser un concepto de éxito, pero 
a la vez son polémicos, ya que, aunque se consideran parte de los ideales jurídicos políticos actuales, aparecen insatisfactoriamente desarrollados y relegados. Indica que las migraciones traen la complejidad cultural de los países, por lo que ya no se pueden atender solo los derechos fundamentales clásicos de las libertades de expresión, reunión, asociación o de la igualdad.

En igual forma se revisa el trabajo de Symonides (s. f), relacionado también con esta temática, el cual plantea que los derechos culturales son los menos desarrollados, necesitan más elucidación, clasificación y fortalecimiento. Esto, por cuanto, el problema se vincula a que estos derechos son abandonados o subestimados dentro de los derechos humanos.

El investigador manifiesta que este descuido puede advertirse en el hecho de que aunque los derechos culturales suelen enumerarse con los derechos económicos y sociales (a los que sí se les presta interés), los primeros reciben menos atención y son olvidados. Inclusive en variadas ocasiones, se limitan a mencionar solo el derecho a la educación, ya que los culturales no son objeto de debate.

Además, Symonides (s. f.) comenta sobre la oposición durante la Conferencia de San Francisco, para que estos derechos se incluyeran en la Carta de las Naciones Unidas. Asimismo se refiere a la no mención de los Derechos Culturales en la Declaración Universal de Derechos Humanos de las personas pertenecientes a minorías, y solo se reconocen en el Artículo 27 del Pacto Internacional de Derechos Civiles y Políticos en 1996.

\section{Los derechos humanos (DDHH) de las personas con discapacidad}

Al respecto se revisa el documento de Quinn, Degener, Bruce, Burke, Castellino, Kenna, Kilkelly y Quinlivan (2002), que trata del uso actual y las posibilidades futuras de los instrumentos en derechos humanos a partir de la discapacidad. El estudio está conformado por tres partes en las cuales se resalta la importancia de los DDHH en esta población, la invisibilidad en las disposiciones de los instrumentos internacionales sobre discapacidad y su necesidad de hacerlas perceptibles. También se evalúa el uso actual de cada uno de los seis instrumentos de la ONU en el contexto de la discapacidad, revisando la participación y la libertad, a partir de lo establecido en el Pacto Internacional de Derechos Civiles y Políticos PIDCP, pues según Quinn, et al. (2002, p. 3), "la máxima aspiración de las personas con discapacidad es tener acceso a los mismos derechos y responsabilidades cívicas que las demás personas", ya que en muchos Estados Partes se considera la discapacidad como un asunto meramente de bienestar social.

Por otra parte, se examina la ética de la justicia social, en donde se enfatiza el disfrute de la libertad de las personas con discapacidad, respetando los derechos económicos, sociales y culturales. Además, se revisa el tema de la tortura, tratos crueles y degradantes que se aplican a esta población, y la doble discriminación contra la mujer, pues se tiende a considerar que estas personas no pertenecen a ninguno de los sexos. El estudio de Quinn et al. (2002) dedica un capítulo de manera especial al análisis de la Convención de Derechos del Niño (CDN) en relación con discapacidad, también hace referencia a grupos raciales y otros grupos minoritarios.

La última parte del trabajo de Quinn et al. (2002) se refiere a observaciones y recomendaciones relacionadas con el uso futuro del sistema de Derechos Humanos de la 
Organización de las Naciones Unidas en el contexto de discapacidad, sugerencias a los Estados Partes para que informen, de manera periódica, sobre la situación de derechos de las personas con discapacidad y, además, piden nombrar a personas con discapacidad como miembros de las organizaciones encargadas de velar por la aplicación de los seis tratados de la ONU.

Asímismo Quinn et al. (2002) reafirman el compromiso de la Oficina del Alto Comisionado de los Derechos Humanos OACDH en relación con la discapacidad, y opinan sobre la urgencia de nombrar un relator o relatora especial sobre los derechos humanos de las personas con discapacidad, y crear un foro o grupo de trabajo con las instituciones nacionales de Derechos Humanos, que permita intercambiar experiencias sobre la temática en cuestión. De igual manera, apoyan la resolución del 2001 de la Asamblea General, donde se establece un comité especial internacional, para que todos los Estados Miembros de la ONU protejan y promuevan los derechos de las personas con discapacidad.

Otro documento que se revisa es el de Lauzurika, Dávila y Naya (2009), producto de una investigación financiada por el Ministerio de Educación y Ciencia de Navarra, en el cual basan sus planteamientos a partir de una educación inclusiva y, por ende, encierra también a las personas con discapacidad. $\mathrm{Al}$ respecto comentan que en el momento del trabajo existen entre 120 y 150 millones de niños y niñas en el mundo que tienen alguna discapacidad, y de los cuales solo el $2 \%$ tiene acceso a la educación, propiciándose así la exclusión social y asumiendo, al mismo tiempo, que quien ofrece impedimentos para su incorporación son las actitudes y barreras que ofrece la sociedad y no la discapacidad misma.

Por tal razón, Lauzurika et al. (2009) consideran que lo importante es garantizar el ejercicio de sus derechos en condiciones de igualdad, la no discriminación o la acción positiva a partir del ordenamiento jurídico internacional. Además indican que la escuela especial se encargó de establecer identidades exclusivas entre las personas que no existían, y dio como resultado un sistema paralelo de educación; en el entendido de que se trata de un único sistema educativo donde toda la población estudiantil despliega su potencial.

Partiendo de estas premisas, Lauzurika et al. (2009) hacen un análisis de algunos instrumentos internacionales y sus artículos específicos sobre educación y discapacidad, entre ellos: la Convención de los Derechos del Niño (CDN), la Convención Interamericana para la eliminación de todas las formas de discriminación contra las personas con discapacidad, y la Convención sobre los Derechos de las personas con discapacidad. Además en su estudio presentan un resumen de la implementación del derecho a la educación de la niñez en América Latina, a partir de los informes de los Estados Partes, y aunque indican que en ellos se incluyen a las personas con discapacidad y la unificación de la educación especial al sistema educativo general, la situación real en los distintos países es otra. En este sentido, mencionan que "la deuda externa, los conflictos políticos y sociales de América Latina, representan situaciones negativas para el cumplimiento de derechos" (Lauzurika et al., 2009, p. 158) y, por lo tanto, en este panorama se circunscribe la educación.

\section{Comentarios, comparaciones y contrastes}

A partir de los planteamientos expuestos, se pueden establecer algunas ideas que permiten reflexionar sobre la temática en estudio. Así, por ejemplo, surgen tres trabajos con ideas similares 
en cuanto a la interiorización musical: es el caso de Arguedas (2003), quien considera que la improvisación representa una forma de hacer música mediante procedimientos que promueven la experimentación, el aspecto lúdico, la espontaneidad, la expresión y la creatividad de las personas, al utilizar objetos, instrumentos musicales, sonidos corporales o la voz humana. Al mismo tiempo, en otro estudio, esta investigadora opina que es necesario propiciar una práctica pedagógica vivencial y significativa para la población estudiantil, mediante experiencias que estimulen cualidades artísticas, y el acceso de estrategias interactivas y novedosas para abordar las distintas áreas de la educación general básica (Arguedas, 2006). Y en igual forma Jiménez (2009) apoya la idea de que la música es sinónimo de integración curricular a nivel de educación preescolar, al esbozar una metodología participativa que lleva a la práctica con docentes y posteriormente con población infantil.

Asimismo Chavarría y Orozco (2006), haciendo énfasis en las inteligencias múltiples y por lo tanto a la musical, analizan el poder transformador de la educación como agente de cambio, donde desde la pedagogía crítica es necesario revisar las normas y rutinas implícitas en el currículo oculto en los procesos de enseñanza y aprendizaje, decodificando lo cotidiano desde el papel de intérpretes culturales, para brindar una sociedad más humanitaria y en constante evolución social.

También Carballo (2006), desde esta misma temática, hace referencia a la importancia de estimular la inteligencia musical, la cual conlleva a la participación de las otras inteligencias de manera implícita. Fernández (2007), de igual manera, establece la importancia de vencer retos a partir de las posibilidades y limitaciones de cada quien, y exalta la participación sin exclusión, pues considera que la resiliencia representa la posibilidad de cambio a partir del reconocimiento de la realidad adversa, donde una de las formas para esa transformación es mediante la expresión artística de cada persona.

Además, desde la expresión artística y el desarrollo humano, Poltronieri (1998) destaca el llamado hacia la calidad, para reivindicar la creatividad y la perspectiva crítica en el trabajo cotidiano de los centros infantiles a partir de las necesidades cognoscitivas, emocionales y sociales de su población. De igual manera y haciendo referencia al desarrollo creativo, Montoya y Sánchez (2000), y Arguedas (2004a) exaltan la importancia de la expresión corporal como parte de las estrategias didácticas a nivel preescolar y escolar, ya que las experiencias que de ellas emanan son aceptadas por la niñez con gran beneplácito, pues le ayudan a descubrir su subjetividad y el disfrute de la recreación.

Igualmente Arguedas, Castro y García (2006), y Arguedas y Castro (2009) establecen propuestas de actividades artísticas al currículo escolar, en donde la música se convierte en un insumo para integrar múltiples situaciones a la experiencia educativa, las cuales permiten enriquecer el modelo pedagógico que se implemente en el aula en forma creativa y novedosa.

Conjuntamente, en el tema de los derechos humanos de las personas con discapacidad, Herrera (2002) comenta que una sociedad de derechos es aquella donde se respeta la accesibilidad y las necesidades de todas las personas, no existe la discriminación y permite la verdadera equiparación de oportunidades; por lo que considera obligatorio el cumplimiento de la Ley 7600, así como la necesidad de lucha por las políticas sociales.

Por otra parte, teniendo en cuenta las investigaciones internacionales relacionadas con la práctica pedagógica musical, Delgado (2008) considera necesario llevar a cabo un conjunto 
de actividades musicales que preparen a la población infantil para apreciarlas, gozar de estas experiencias estéticas y, de esta manera, el personal docente de esta área contribuye a formar el gusto musical de sus estudiantes potenciando la creatividad.

Además, llama la atención que Sihana y Pechin (2002), en la categoría de educación musical y currículo escolar opinan que es importante poner en práctica estrategias novedosas y no cargadas de teoría. En este sentido, Rusinek (2004) considera que el currículo muchas veces se limita a ofrecer información verbal de la música, lo que resulta totalmente inapropiado para la educación, pues no ofrece un planteamiento activo mediante el desarrollo de habilidades. Esboza la necesidad de cuestionarse lo que cada persona asimila de acuerdo con su edad, cómo se relacionan los conceptos con los eventos musicales y de qué manera dichos conceptos no van a ser olvidados.

Por eso Cantón y Fabián (2010), en un planteamiento similar, enfatizan en la necesidad de una adecuada formación musical del equipo docente, que no esté fundamentada tan solo en una especialidad instrumental, ya que este contexto desemboca en una repetición de la manera que se formó desde los conservatorios; todo lo contrario, debe implementar estrategias acordes a las necesidades de sus estudiantes.

Pero al mismo tiempo, los datos obtenidos en este estudio plantean otras posiciones interesantes en relación con la expresión artística y, por ende, musical. Es el caso de Palacios (2006), quien opina que en la sociedad moderna el arte en general no tiene razón de ser y ocupa un lugar frágil en el currículo escolar, pues diferentes aspectos sociales y económicos impiden su valoración en el ámbito educativo. Cuando, por el contrario, la escuela debe ser el espacio para ofrecer la oportunidad de promover múltiples formas de inteligencias, donde la población estudiantil pueda elegir aquella que corresponda a sus aptitudes y satisfaga plenamente sus intereses. Al respecto, para apoyar su teoría se fundamenta en autores como Arnheim, Gardner, Goodman, Cassirer, Langer y Eisner, y considera la música como aquella que ocupa un lugar importante dentro de los lenguajes artísticos.

Asimismo Poch (2002), Purroy y Pérez (2002), Lagos (2004), Gutiérrez, Ibáñez y Prieto (2011) y Pineda y Pérez (2011) presentan en sus trabajos opiniones similares en relación con el uso de la musicoterapia en personas con síndrome de Down, al indicar que mediante ella se obtiene el logro de objetivos socioafectivos, cognitivos y psicomotores, tal y como lo confirma la siguiente cita:

La musicoterapia puede influir de manera directa o indirecta, sobre el bienestar emocional (gratificación, falta de estrés, autoconcepto); las relaciones interpersonales (interacción, relaciones, apoyo emocional; el desarrollo personal (educación, competencia personal, actuación) la autodeterminación (autonomía, valores personales, elección); la inclusión social (integración en la comunidad, apoyo social) y los derechos (igualdad). (Gutiérrez, Ibáñez y Prieto, 2011, p. 30)

Al mismo tiempo Ferrándiz, Prieto, Bermejo y Ferrando (2006) y Luz de Luca (s. f.), concuerdan en la importancia de estimular las inteligencias múltiples en beneficio del desarrollo integral de la persona y de acuerdo con sus necesidades particulares. Por tal motivo, 
Luz de Luca (s. f.) prueba, con gran variedad de actividades: lee toda clase de bibliografía y prepara un escrito para compartirlo con docentes que se encuentran ante situaciones similares y no tienen un equipo de profesionales a quien acudir en busca de ayuda.

Además, es interesante mencionar que Green (2001) trata el desarrollo humano mediante la música y la integración de las artes, ya que considera que se solventan carencias de quienes participan en la experiencia. Igualmente establece distintas percepciones sobre la expresión artística, entre las que se encuentran la creatividad, la comunicación, el entretenimiento, la identificación y la diferencia, entre otras.

Mientras, Sanfeliu y Caireta (2005) proponen la expresión "música por la paz positiva", entendida esta como aquella que ayuda a conseguir la armonía entre las personas respetando los derechos humanos. Es así que conciben la educación por la paz como un proceso dinámico, continuo y permanente, que a través de enfoques socioafectivos y problematizadores, pretende desarrollar una nueva cultura que ayude a las personas a observar críticamente la realidad para enfrentarla y actuar.

Por su parte, Pérez (2008) considera al personal docente como un agente potencial del proceso didáctico, el cual debe promover la calidad educativa y el desarrollo integral. Es decir, que las acciones educativas que promueva, faciliten la interrelación de saberes en beneficio del desarrollo humano de la población estudiantil, sin dejar de lado la lúdica creativa y la vivencia musical.

Ahora bien, como un aporte al tema de enfoque de derechos y expresión artística, OlsonHorswill (2005) expresa que el arte une a las personas, tal y como lo plantea la teoría de Dewey, ya que permite expresar sus críticas, sentimientos e identidades, mediante líneas, colores, textos, formas y texturas, articulándose de esta manera el espíritu universal de los derechos humanos. No obstante, Prieto de Pedro (2008) externa su preocupación en cuanto a los derechos culturales, pues aunque considera que son de gran importancia para las personas como parte de su identidad y crecimiento artístico, en muchos casos son minimizados al negarse por completo su validez.

Igualmente conviene conocer a Symonides (s. f) en una posición análoga, al manifestar que estos derechos suelen calificarse como "categoría subdesarrollada" de derechos humanos, sugiriendo que en comparación con los derechos civiles, políticos, económicos y sociales, los derechos culturales son los que menos se llevan a la práctica en relación con los aspectos jurídicos y la posibilidad de hacerlos respetar. Cabe rescatar que dentro de las aportaciones que ofrecen los trabajos de Olson-Horswill y Symonides, se destaca la necesidad de hacer conciencia social sobre la importancia de los derechos culturales dentro de los derechos humanos, pues aunque se han adoptado directrices sobre la temática, es imprescindible darle un mayor auge y aplicarse sin distinción de ninguna clase, pues en reiteradas ocasiones en poblaciones minoritarias, se irrespetan e invisibilizan.

En igual situación se encuentran los derechos humanos de las personas con discapacidad, de ahí que es interesante comentar la posición de Quinn et al. (2002), pues opinan que esta población debe tratarse como sujetos y no como objetos y, por lo tanto, es necesario ubicar la problemática fuera de la persona con discapacidad, y tratar la no discriminación, la igualdad $\mathrm{y}$ el disfrute de todos sus derechos, como tema central en todos los sistemas sociales y 
económicos, teniendo en cuenta, además, la dignidad, la autonomía, y la solidaridad. Por tal motivo hacen un señalamiento a los instrumentos de la ONU en el marco de la discapacidad y sugieren acciones importantes a seguir entre los Estados miembros de esta entidad.

Por otra parte, en un contexto similar, Lauzurika, et al. (2009) defienden la necesidad de garantizar el cumplimiento de derechos a través de la puesta en práctica, donde la educación constituya un elemento relevante para la integración social de las personas con discapacidad. No obstante mencionan "solo Salvador, Panamá, Paraguay, Perú, República Dominicana y Venzuela, contemplan de manera específica en su texto constitucional, la inclusión de estos niños y niñas más allá de la simple integración”. (Lauzurika, et al., 2009, p. 157). Igualmente expresan que países como Argentina, Brasil, Chile, Salvador, Honduras, México, Panamá y Paraguay poseen cifras altas de niños, niñas y personas adolescentes con discapacidad con limitado acceso a la educación.

Ahora bien, después de conocer los planteamientos antes citados, se considera oportuno también externar el posicionamiento de quien escribe el presente artículo, ya que posee puntos en común con los del grupo de autores que se mencionan; he aquí algunos de ellos:

La educación musical es sinónimo de integración curricular al actuar como eje transversal en el quehacer educativo, pues propicia metodologías en donde se interrelacionan saberes que invitan a participar de manera creativa y lúdica. Esto implica aprendizajes significativos y de esparcimiento, que conllevan a una educación de calidad como parte de los derechos de la niñez y de la adolescencia.

Es así como la música constituye un elemento ideal para lograr el disfrute de la recreación, al permitir la expresión de emociones y sentimientos. Al mismo tiempo estimula otras inteligencias del ser humano, pues la música es matemática, representa un lenguaje, implica un comportamiento cinestésico o corporal, y posee un componente intrapersonal e interpersonal, sin dejar de lado la expresión espiritual de las personas.

En este sentido, el hogar y el centro educativo son espacios ideales para promover el desarrollo humano mediante la música y la integración de las artes con otras áreas curriculares. La educación musical representa un planteamiento activo para motivar el desarrollo cognitivo, socioafectivo y psicomotor; en otras palabras, estimula el desarrollo integral de la población infantil y adolescente.

Por otra parte, favorece la interacción de cada individuo consigo mismo, con los demás, con los objetos y la naturaleza; de esta manera promueve el compañerismo, el trabajo en equipo, y el respeto a la opinión y al ambiente entre otros valores, respetando los derechos humanos e incentivándose lo que Sanfeliu y Caireta (2005) denominan "música por la paz positiva".

Al mismo tiempo, la educación musical debe tomar en cuenta las necesidades de la población infantil y adolescente, pero además los intereses que tengan, pues de esta forma se exalta la autonomía y el respeto a los derechos de las personas.

No obstante esta participación musical y desarrollo de la sensibilidad artística tiene que darse sin exclusión alguna, respetando la legislación existente en Derechos Humanos de Niñez y Adolescencia, y en el caso que ocupa este artículo, a las personas en condiciones asociadas o no a discapacidad. De esta manera se tiene presente la autonomía, la solidaridad 
y la dignidad de las personas, trayendo como consecuencia la no discriminación y la igual de oportunidades, así como también el acatamiento a los derechos culturales.

Por lo tanto, el recuento de las indagaciones antes mencionadas permite mostrar diversas posiciones de investigadoras e investigadores que reflejan puntos de encuentro o de contraste con la temática de interés. Por otro lado, surge la necesidad de implementar vivencias musicales significativas que vayan más allá de los aspectos teóricos y memorísticos; por el contrario, deben brindarse espacios donde se estimule la sensibilidad y el desarrollo creativo para todas las personas sin ninguna clase de discriminación; en los que se promueva el disfrute de la vivencia sonora, la cual puede integrarse a otras áreas del saber teniendo en cuenta la integralidad de la persona y los derechos humanos.

Asimismo, es necesario realizar en nuestro país investigaciones en donde se articule la educación musical con el síndrome de Down, así como también surge la urgencia de llevar a cabo indagaciones donde se integren el enfoque de derechos y la expresión artística (de manera especial en relación con la enseñanza de la música, como parte de la investigación que dio origen a este artículo), pues en ambos casos al efectuar esta revisión bibliográfica, los trabajos que se encuentran son escasos.

\section{Conclusiones}

Esta recolección bibliográfica se lleva a cabo mediante la búsqueda de diversos documentos relacionados con la temática de interés, entre ellos: libros, revistas, artículos e investigaciones, que se obtienen por vía internet o de manera física. Posteriormente se hace una lectura de ellos y se analizan, con la finalidad de que la información sea pertinente y se convierta en insumos importantes para el enriquecimiento de la investigación "Análisis del modelo pedagógico musical que utilizan los Programas Especiales del Sistema Nacional de Educación Musical del Ministerio de Cultura y Juventud, en relación con las necesidades e intereses de sus estudiantes desde el enfoque de derechos humanos”.

De esta manera, los trabajos consultados en el momento en que se realiza el presente estado del arte o estado de la cuestión brindan una perspectiva del binomio derechos humanos y educación musical. Al mismo tiempo, mediante la síntesis analítica se puede establecer una valoración de los resultados encontrados sobre la temática en estudio, y relacionar los procesos metodológicos en que participa la población estudiantil de los Programas Especiales del SINEM, institución de interés en la investigación que da origen a este artículo. Como consecuencia, se establecen las siguientes categorías afines con el trabajo:

- Formación en valores: la tolerancia, el compañerismo, la convivencia y el trabajo en equipo.

- Sensibilización artística en poblaciones diversas.

- Modificación de conductas biológicas, sociales, psicológicas y culturales como parte del desarrollo humano.

- Estimulación de las inteligencias múltiples.

- Vivencia, interiorización y creación musical. 
- Estimulación cognitiva, psicomotora y socioafectiva de la población estudiantil.

- La música como elemento de comunicación.

- Lúdica y creatividad en el proceso educativo.

- Calidad en la enseñanza musical.

- Educación musical inclusiva.

- Integración de la música y la expresión corporal.

- La expresión artística y el currículo escolar.

- Música como elemento de paz.

- Música y diversidad de expresión cultural.

- La discapacidad y los derechos humanos.

Por otra parte, se estima que en la totalidad de estas indagaciones, de preferencia se trata el tema musical como una de las inteligencias múltiples. Asimismo, es interesante rescatar que el país con mayores aportes al tema en estudio es España, aunque también se obtiene información de Estados Unidos, Colombia, Argentina y Venezuela. Además, se consigue información con respecto a discapacidad y derechos humanos, pero no integrado a la educación musical.

De Costa Rica se rescata que hay pocas personas que han incursionado en estudios relacionados con esta temática, entre ellos se consiguen trece trabajos, de los cuales la proponente de la investigación, es al mismo tiempo una de las autoras de dichas indagaciones, las cuales ha desarrollado en diversos proyectos de Acción Social e Investigación de la Universidad de Costa Rica, con población infantil y docentes de educación preescolar, primaria y secundaria de diferentes contextos educativos costarricenses.

Asimismo, la educación musical y su relación con derechos humanos de niñez y adolescencia, es un tema no investigado en Costa Rica a nivel de tesis tanto de grado como de posgrado. En este sentido, representa una realidad lamentable y contradictoria para la niñez y las personas adolescentes de nuestro país, pues tal y como lo expresa el Consejo Nacional de Niñez y Adolescencia (CNNA, 2009), desde la Política Nacional de Niñez y Adolescencia PNNA, se enfatiza en la necesidad de la igualdad de oportunidades, la equidad, la autonomía progresiva, la discriminación positiva, la promoción de la recreación, y el fortalecimiento de una educación artística y estética.

Si bien es cierto, existen documentos a nivel nacional relacionados con la educación musical, desde la perspectiva de derechos vinculada a la expresión artística (de manera específica musical), las investigaciones no tienen el componente de derechos humanos de niñez y adolescencia. Llama la atención la ausencia de trabajos de esta índole, pues en la actualidad representa una necesidad urgente a nivel nacional y mundial por la situación social compleja que viven estos grupos etáreos producto de la pobreza, las drogas, la callejización, la deserción escolar, la desintegración familiar, y la explotación sexual comercial, entre otros. 
En estos contextos, la educación musical puede convertirse en una opción significativa en la restitución de derechos de la población infantil y adolescente así como de su cumplimiento y garantía, y al mismo tiempo parte de los derechos culturales. Es conveniente también indicar, que aunque este rubro de derechos se ubica como de segundad generación (debido a que fueron los segundos en ser protegidos por las constituciones políticas), no implica una menor importancia en su aplicación en niñez y adolescencia, pues desde el inicio fueron reconocidos por convenios internacionales, y son relevantes para el desarrollo integral de las personas.

Al mismo tiempo, se debe tener presente que la música favorece el desarrollo de las personas desde los componentes socioafectivos, psicomotores y cognitivos, estimula la expresión de sentimientos, estados de ánimo e inquietudes, permite reforzar la autoimagen y la autoestima, al igual que facilita el crecimiento espiritual mediante la incentivación de la creatividad y la sensibilidad; es decir, representa una posibilidad para devolver condiciones en donde se han violentado los derechos humanos. Como consecuencia, los planteamientos antes mencionados permiten justificar la importancia de la música dentro de los derechos culturales y por ende, de los derechos humanos, ya que son imprescindibles en la formación de la población infantil y adolescente como parte de su desarrollo integral.

Por tales razones, insto a quienes desean indagar en este tópico, a que investiguen y escriban sus planteamientos desde la vivencia musical, y su implicación en el desarrollo de la población infantil y adolescente en condiciones asociadas o no a la discapacidad y el enfoque de derechos humanos, ya que esta interrelación brinda un complemento valioso para la formación de las personas, y desarrolla la sensibilidad y el respeto a las necesidades e intereses particulares. De esta manera, estos aportes se convierten en herramientas importantes a nivel de familia, de centros educativos y de comunidades, para el bien común de la sociedad y, por consiguiente, de la humanidad.

\section{Referencias}

Arguedas, C. (2003). La improvisación musical y el currículo escolar. Revista Electrónica Actualidades Investigativas en Educación, 3(2), 1-21. Recuperado de http://revista.inie. ucr.ac.cr/uploads/tx magazine/musical.pdf

Arguedas, C. (2004a). La expresión corporal y la transversalidad a partir de la expresión artística. Revista Educación, 28(1), 123-131. Doi: http://dx.doi.org/10.15517/revedu. $\underline{\mathrm{v} 28 \mathrm{i} 1.2830}$

Arguedas, C. (2004b). La expresión musical y el currículo escolar. Revista Educación, 28(1), 111-122. Doi: http://dx.doi.org/10.15517/revedu.v28i1.2823

Arguedas, C. (2006).Vivencias de la expresión musical: Áreas y estrategias metodológicas. Revista Electrónica Actualidades Investigativas en Educación, 6(3), 1-30. Recuperado de http://revista.inie.ucr.ac.cr/uploads/tx magazine/vivencias.pdf 
Arguedas, C. (2009). Música y expresión corporal en los procesos de enseñanza y aprendizaje del inglés, español y francés. Revista Electrónica Actualidades Investigativas en Educación, 9(1), 1-30. Recuperado de http://revista.inie.ucr.ac.cr/uploads/tx magazine/ musica.pdf

Arguedas, C. Castro, J. y García, J. (2006). La expresión artística: Un medio para construir procesos pedagógicos significativos en el aula. Actividades Costa Rica: Gráficas ACTIGRAF, S.A.

Arguedas, C. y Castro, J. (2009). Las artes plásticas, la música y la expresión corporal... más allá del aula. San José, Costa Rica: Colección Yigüirro. Instituto de Investigación en Educación (INIE).

Bonilla, Y. García J. y Ledezma, E. (2001). La expresión corporal y al música en el currículo de educación inicial. (Memoria del Seminario de Graduación para optar por el título de Licenciatura de Ciencias de la Educación con énfasis en Educación Preescolar), Universidad de Costa Rica, San José, Costa Rica.

Cantón, I. y Fabián, G. (2010). Del currículum musical prescrito al currículum musical práctico en el aula de educación primaria. Bordón Revista de Pedagog 62(2), 109-126. Recuperado de http://dialnet.unirioja.es/servlet/articulo?codigo=3235628

Carballo, S. (2006). Educación de la expresión de la sexualidad y la inteligencia emocional en niñas, niños y adolescentes con derechos. Revista electrónica Actualidades Investigativas en Educación, 6(3), 1-30. Recuperado de http://revista.inie.ucr.ac.cr/ uploads/tx magazine/musica.pdf

Chavarría, M. C. y Orozco, C. (2006). Ecoanálisis como puerta de entrada a la decodificación de lo cotidiano: Hacia una educación posible. Revista electrónica Actualidades Investigativas en Educación, 6(3), 1-35. Recuperado de http://revista.inie.ucr.ac.cr/uploads/tx magazine/ ecoanali.pdf

Consejo Nacional de la Niñez y la Adolescencia (CNNA). (2009). Política nacional para la niñez y la adolescencia en Costa Rica 2009-2021 MIDEPLAN. PANI. UNICEF. San José, Costa Rica: Autor.

Delgado, María de los Ángeles. (2008). ¿Por qué estudiar música en la escuela? Propiedades de la música, desarrollo integral y poder formativo. Enfoques Educativos, 3(1). 23-36. Recuperado de http://www.enfoqueseducativos.es/ ciencia/ciencia 3.pdf\#page=23

Fernández, M. (2007). El reconocimiento de las necesidades educativas de los niños y niñas 
con talento artístico (Tesis de licenciatura). Universidad de Costa Rica. San José, Costa Rica.

Ferrándiz, C., Prieto, M. D., Bermejo, M. R. y Ferrando, M. (2006). Fundamentos psicopedagógicos de las inteligencias múltiples. Revista Española de Pedagogía, 64(233), 5-20. Recuperado de http://www.academia.edu/2452898/Fundamentos psicopedagogicos de las inteligencias multiples

García, J. Castro, J. y Arguedas, C. (2003). Informe final. La expresión artística un recurso heurístico para la construcción de procesos pedagógicos en el aula desde una perspectiva de género. Facultad de Educación. Universidad de Costa Rica. San José: Instituto de Investigación para el Mejoramiento de la Educación Costarricense (IIMEC).

Green, J. (2001). Social application of the arts. Making a difference through Art [Aplicación social de las artes. Haciendo una diferencia a través del arte] United States: Degree of Doctor of Philosophy University of Washington.

Gutiérrez, P. Ibáñez, P. y Prieto, S. (2011). Yo quiero ser Marifé de Triana. Experiencia para el desarrollo de la inteligencia emocional a través de la musicoterapia. Tejuelo: Didáctica de la Lengua y la Literatura, 13, 24-46. Recuperado de http://dialnet.unirioja.es/servlet/ busquedadoc?t=Guti\%C $3 \%$ A9rrez $\% 2 \mathrm{C}+$ Prudencia. $+\mathrm{Ib} \% \mathrm{C} 3 \% \mathrm{~A} 1 \% \mathrm{C} 3 \% \mathrm{~B} 1 \mathrm{ez} \% 2 \mathrm{C}+$ Patrici a. + Prieto + Sara. $++\& d b=1 \&$ td $=$ todo

Herrera, N. (2002). Visión crítica de la legislación sobre la discapacidad en Costa Rica: Un acercamiento desde los derechos humanos. Revista ESPIGA, 3(6), 51-64. Recuperado de http://estatico.uned.ac.cr/sociales/publicaciones/documents/06ESPIGA6.pdf

Jiménez, M. (2009). La educación musical como estrategia facilitadora del currículo para el ciclo materno infantil (grupo de interactivo ii) y ciclo de transición de la educación inicial: Una propuesta metodológica (Informe final de licenciatura). Universidad de Costa Rica, San José, Costa Rica.

Lagos, E. (2004). Musicoterapia en niños con síndrome de Down: La importancia del vínculo en la creatividad y desarrollo emocional (Monografía). Universidad de Chile. Recuperado de http://www.tesis.uchile.cl/tesis/uchile/2004/lagos e/html/index-frames.html

Lauzurika, A., Dávila, P., y Naya, L. (2009). El derecho a la educación de las personas con discapacidad: Una aproximación desde América Latina, en los últimos quince años. En M. Reyes y S. Conejero (Coords.), El largo camino hacia una educación inclusiva: La educación especial y social del siglo XIX a nuestros días. XV Coloquio de Historia de la Educación, Pamplona-Iruñea, (pp. 147-160). España: Universidad Pública de Navarra. 
Luz de Luca, S. (s. f.) El docente y las inteligencias múltiples. Revista Iberoamericana de Educación, 1-12. Recuperado de http://www.rieoei.org/deloslectores/616Luca.PDF

Montero, F. (2004). Los derechos culturales: Un acercamiento a su contenido programático y aplicabilidad normativa. Cuadernos de Antropología, 14, 47-59.

Montoya, M. y Sánchez, A. (2000). Lineamientos metodológicos para estimular la creatividad mediante la expresión corporal en el niño preescolar de 5 años y 6 meses a 6 años 6 meses. (Memoria de licenciatura). Universidad de Costa Rica, San José, Costa Rica.

Mora, D. (2001). Efectos de la músicoterapia y la danzaterapia en el comportamiento no verbal de personas con discapacidad múltiple severa (Tesis de maestría). Universidad de Costa Rica, San José, Costa Rica.

Olson-Horswill, L. (2005). Learning the arts and Human Rights. An extraordinary connection [Aprendiendo las artes y los derechos humanos. Una extraordinaria conexión (Tesis de doctorado). United States: University of Idaho.

Palacios, L. (2006). El valor del arte en el proceso educativo. Reencuentro, 46, 2-22. Recuperado de http://biblioteca.clacso.edu.ar/subida/Mexico/dcsh-uam-x/20121122103148/valor.pdf

Pérez, M. A. (2008). Evolución de la práctica pedagógica como dispositivo escolar y discursivo en la educación artística - musical. latinoam.estud.educ., 4(1) 49 -61. Recuperado de http://latinoamericana.ucaldas.edu.co/downloads/Latinoamericana4(1) 4.pdf

Pineda, E. y Pérez, Y. (2011). Musicoterapia aplicada a niños con síndrome de Down. Revista Cubana de Pediatría, 83(1),142-148. Recuperado de http://scielo.sld.cu/scielo. php?pid=S0034-75312011000200003\&script=sci arttext

Poch, S. (2002). Introducción a la musicoterapia. En Confederación ASPACE, Federación Española del Síndrome de Down y Confederación Autismo España (Eds.) , Programa de Formación para Mediadores en Musicoterapia y Discapacidad. Libro de ponencias (pp. 5-48). Madrid: Editores. Recuperado de http://sid.usal.es/libros/ discapacidad/6823/8-4-2/programa-de-formacion-para-mediadores-en-musicoterapia-ydiscapacidad-musicoterapia-2002.aspx

Poltronieri, P. (1998). Evaluación del impacto de un proceso de capacitación y reestructuración del ambiente educativo, en la calidad de la atención a los niños/as en centros infantiles de atención integral (CEN-CINAI) (Tesis de maestría). Universidad de Costa Rica, San José, Costa Rica. 
Prieto de Pedro, J. (2008). Derechos culturales, el hijo pródigo de los Derechos Humanos. Revista Crítica, 952, 19-23. Recuperado de http://www.uned.es/ca-campo-degibraltar/ TABLON/derecho/Derechos\%20culturales\%20el\%20hijo\%20prodigo\%20de\%20los\%20 Derechos\%20Humanos.pdf

Purroy, C. y Pérez, C. (2002). Musicoterapia y síndrome de Down. En Confederación ASPACE, Federación Española del Síndrome de Down y Confederación Autismo España (Eds.), Programa de Formación para Mediadores en Musicoterapia y Discapacidad. Libro de ponencias (pp. 51-75). Madrid: Editores. Recuperado de http://sid.usal.es/libros/ discapacidad/6823/8-4-2/programa-de-formacion-para-mediadores-en-musicoterapia-ydiscapacidad-musicoterapia-2002.aspx

Quinn, G. Degener, T. Bruce, A. Burke, C. Castellino, J. Kenna, P. Kilkelly, U. y Quinlivan, S. (2002). Derechos humanos y discapacidad. Uso actual y posibilidades futuras de los instrumentos de Derechos Humanos de las Naciones Unidas en el contexto de la discapacidad. New York y Ginebra: Organización del Alto Comisionado de los Derechos Humanos OACDH. ONU. Recuperado de http://www2.ohchr.org/spanish/ about/publications/docs/disability.pdf

Riera, L. Sansevero, I. y Lúquez, P. (2008). La cultura democrática en la formación integral de la educación básica. Revista Educación en Valores, 10, 9-20. Recuperado de http:// dialnet.unirioja.es/servlet/articulo?codigo $=3268162$

Rusinek, G. (2004). Aprendizaje musical significativo. Revista electrónica complutencce de investigación en educación musical, 1(5), 1-16. Recuperado de http://www.ucm.es/infol reciem/v1n5.pdf

Sanfeliu, A. y Caireta, M. (2005). La música com a instrument d' educación per a la pau. Quaderns d'educació per a la pau. [La música como un instrumento de educación para la paz. Cuadernos de educación para la paz]. (1-20) Recuperado de http://ibdigital.uib. es/greenstone/collect/cd2/import/escola de cultura de pau/drets humans/monografia/ publicacion010c.pdf

Sihana, M. y Pechin, C. (2002). Representaciones sociales de los/las estudiantes en la formación docente acerca de las áreas música y educación física. Anuario N. 4 . Facultad da Ciencias Humanas - Universidad Nacional de La Pampa. Argentina. (39-50). Recuperado de http://www.biblioteca.unlpam.edu.ar/pubpdf/anuario fch/n04a03franco. pdf

Symonides, J. (s. f.) Derechos culturales: Una categoría descuidada de derechos humanos. Revista Internacional de Ciencias Sociales, 11(5), 1-20. Recuperado de http://www. redinterlocal.org/IMG/pdf Derechos culturales.pdf 